\title{
A First-Principles Study of the Vacancy-Mediated Silver Impurity Diffusion in fcc Nickel
}

Jianguo $\mathrm{Yu}$

September 2019

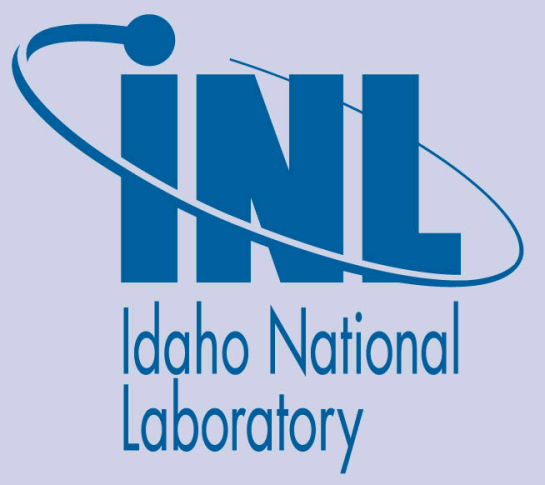




\section{DISCLAIMER}

This information was prepared as an account of work sponsored by an agency of the U.S. Government. Neither the U.S. Government nor any agency thereof, nor any of their employees, makes any warranty, expressed or implied, or assumes any legal liability or responsibility for the accuracy, completeness, or usefulness, of any information, apparatus, product, or process disclosed, or represents that its use would not infringe privately owned rights. References herein to any specific commercial product, process, or service by trade name, trade mark, manufacturer, or otherwise, does not necessarily constitute or imply its endorsement, recommendation, or favoring by the U.S. Government or any agency thereof. The views and opinions of authors expressed herein do not necessarily state or reflect those of the U.S. Government or any agency theof. 


\title{
A First-Principles Study of the Vacancy-Mediated Silver Impurity Diffusion in fcc Nickel
}

\author{
Jianguo $\mathrm{Yu}$
}

September 2019

Idaho National Laboratory Idaho Falls, Idaho 83415

http://www.art.inl.gov

Prepared for the U.S. Department of Energy Office of Nuclear Energy Under DOE Idaho Operations Office

Contract DE-AC07-05ID14517 



\title{
A First-Principles Study of the Vacancy-Mediated Silver Impurity Diffusion in fcc Nickel
}

\author{
INL/EXT-19-55762
}

September 2019

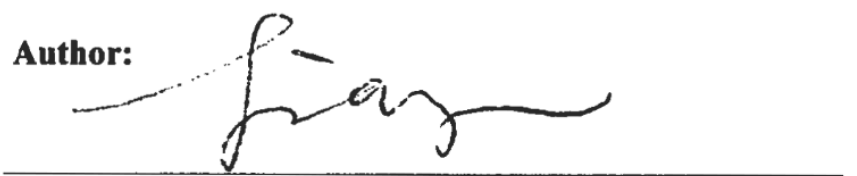

Jianguo Yu

Staff Scientist

Technical Reviewer:

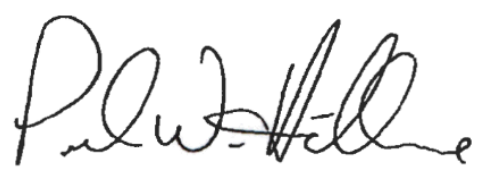

Paul Humricklouse

Staff Scientist

Approved by:

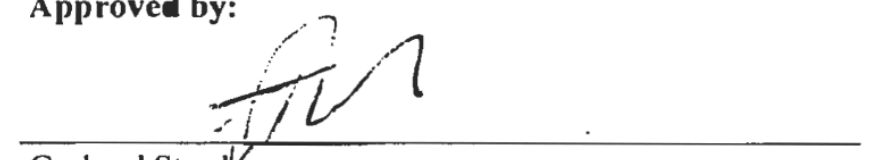

Gerhard Strydom

Co-Director ART NTRD

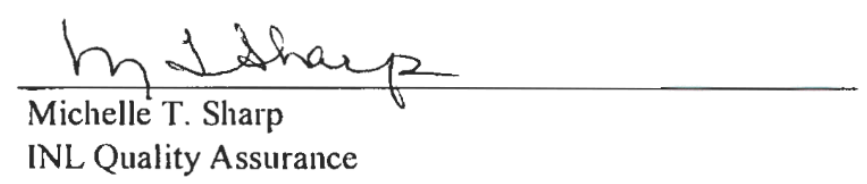

$9 / 12 / 2019$

Date

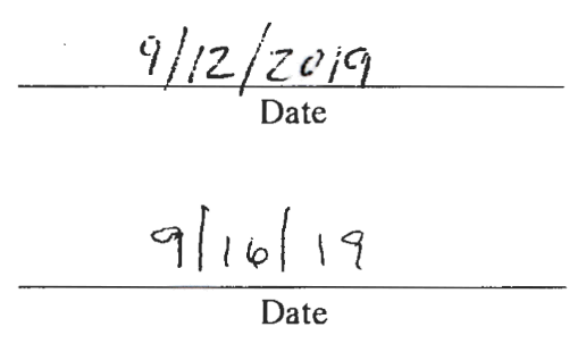





\begin{abstract}
In this report, $a b$ initio techniques in Density Functional Theory, (DFT) are used to calculate the vacancy-mediated self-diffusion coefficient and impurity diffusion coefficient of silver (Ag) in pure fcc nickel (Ni). Prediction of diffusion coefficients is one of the most stringent tests of the ability of DFT calculations, because of the complex interplay of lattice constant, vacancy formation enthalpy, solute-vacancy binding enthalpy, and vibrational frequencies. The Ag diffusion and $\mathrm{Ni}$ self-diffusion coefficients calculated in the present work compare very favorably to previous experimental self-diffusion and $\mathrm{Ag}$ diffusion data. Extension of the methodology to nickel alloys is envisioned in future work.
\end{abstract}




\section{CONTENTS}

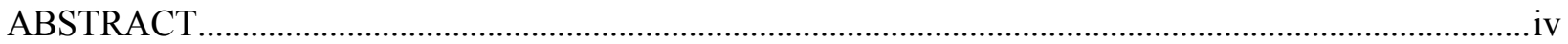

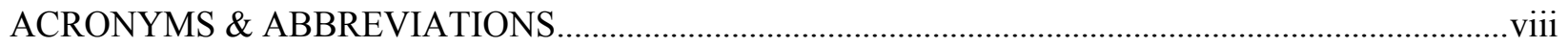

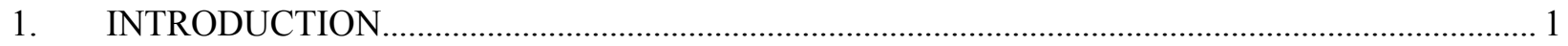

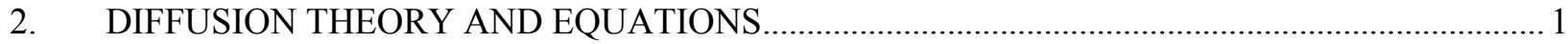

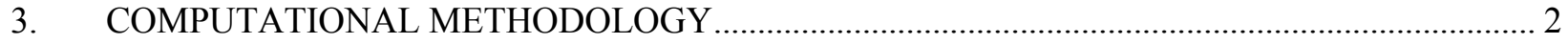

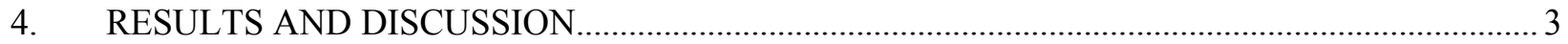

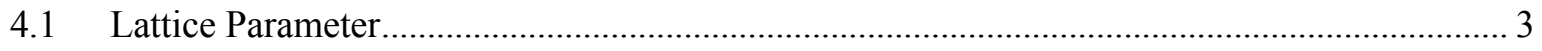

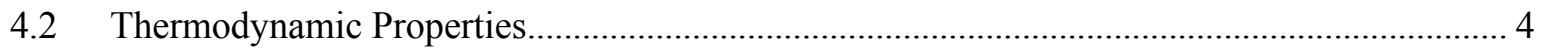

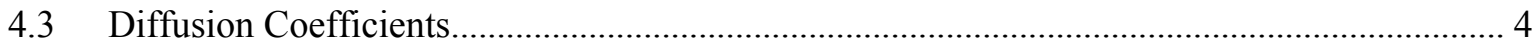

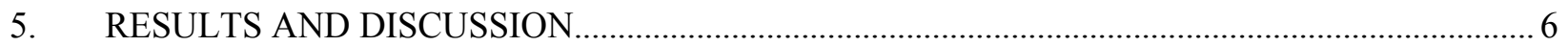

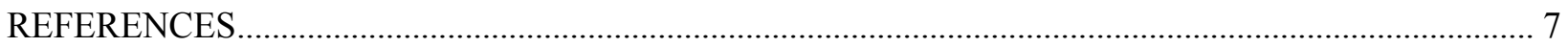

\section{FIGURES}

Figure 1. Coefficients of self-diffusion (top) and vacancy-mediated Ag diffusion (bottom) in pure fcc Ni for two vacancy formation vibrational entropies of $1.8 k_{\mathrm{B}}$ and $0 k_{\mathrm{B}}$, respectively, compared with experimental data. .6

\section{TABLES}

Table 1. Calculated properties from current PBE calculations to, in comparison with, experimental data and other theoretical calculations. 


\section{ACRONYMS \& ABBREVIATIONS}

DFT Density Functional Theory

DFPT Density Functional Perturbation Theory

fcc face-centered cubic

GGA Generalized Gradient Approximation

GW One-body Green's function (G) and dynamically screened Coulomb interaction (W)

HTTR High Temperature Test Reactor

LDA Local Density Approximation

NEB Nudged Elastic Band Method

PAW Projector Augmented Wave

PBE Perdew-Burke-Ernzerhof

TST Transition State Theory

VASP Vienna Ab initio Simulation Package 


\section{A First-Principles Study of the Vacancy-Mediated Silver Impurity Diffusion in fcc Nickel}

\section{INTRODUCTION}

Silver possesses a peculiar ability to escape the silicon carbide layer of tristructural-isotropic (TRISO) fuel used in high-temperature gas reactors (HTGRs). While it is not thought to be a significant contributor to source terms, silver released in this manner may nevertheless be transported throughout the primary circuit. In an HTGR employing a direct Brayton cycle, ${ }^{110 \mathrm{~m}} \mathrm{Ag}$ may deposit on turbine blades, and thus represents a potentially significant contributor to the dose received by personnel performing necessary maintenance on the turbine. If ${ }^{110 \mathrm{~m}} \mathrm{Ag}$ deposited on the surface proceeds to diffuse into the bulk of the turbine blade material, it ceases to be removable contamination, exacerbating the problem. It is therefore of interest to understand the mechanisms of silver diffusion into candidate high-nickel superalloys, in order to inform alloy selection so as to mitigate this issue.

Diffusion is responsible for many different material properties and phenomena, such as diffusional phase transformations, high-temperature creep, and coarsening. One of the most common means of atomic diffusion in crystalline solids is via the vacancy mechanism [1], and vacancies are therefore of fundamental importance to the properties of metals and alloys.

Parameter-free first-principles approaches based on density functional theory (DFT) provide a powerful method for obtaining detailed point defect energetics. They are increasingly used to predict selfand impurity diffusion of elements in pure metals and multi-component alloys [1,2-4]. In this report, we demonstrate the feasibility of combining a thermodynamics integration method with ab initio total energy calculations in order to evaluate thermodynamics properties, namely the impurity coefficient. We demonstrate the efficacy of the methodology by applying it to both self-diffusion in pure fcc nickel (Ni), and impurity diffusion of silver $(\mathrm{Ag})$ in pure fcc $\mathrm{Ni}$, and comparing with experimental data [5-7]. The ultimate objective is to apply similar methods as predictive models for Ag diffusion in high-nickel superalloys, and extension to these more complicated materials is discussed.

\section{DIFFUSION THEORY AND EQUATIONS}

The present work focuses on vacancy-mediated self-diffusion and Ag diffusion in the pure fcc $\mathrm{Ni}$ crystalline solid and calculates the lowest energy diffusion path of an atom between the initial and final states of an elementary atomic jump. Essentially, two processes are occurring. First, the defect, in this case a vacancy or Ag, is formed. Second, a thermally activated jump occurs, where the diffusing atom and the first nearest neighbor vacancy exchange lattice sites, known as vacancy-atom exchange. The macroscopic diffusion coefficient can then be written in terms of microscopic parameters; i.e., the atomic jump distance and jump frequency. In order to compare experimental data directly, one can represent the diffusion coefficient in Arrhenius form [8]:

in which is the prefactor (intercept), is Boltzmann's constant and is the activation energy for diffusion

(slope). The activation energy for diffusion, , is 
the summation of enthalpies of vacancy formation, , and binding with impurity $X$, - and impurity

migration:

The prefactor, , includes the change in the entropy of the lattice vibrations that arises from the constriction of the diffusing atom when it is at the top of the barrier. It may be defined as

where is the jump correlation factor, here equal to 0.7815 [9] for the fcc lattice, is the lattice parameter,

and and are the changes in the vibrational entropies due to the introduction of one vacancy and the

vacancy binding with impurity $\mathrm{X}$ (here, $\mathrm{Ag}$ ). They are related to changes in the vibrational frequencies of atoms surrounding the vacancy or vacancy-impurity pair. The effective jump frequency is , which can be determined according to the harmonic approximation within the transition state theory (TST) [10].

In particular, the effective jump frequency is expressed as the ratio of the product of normal vibration frequencies of the initial state of atomic migration to that of the non-imaginary normal frequencies of the transition state, 
in the initial well and in the activated transition state, respectively, for a system of $N$ atoms. The vacancy

formation energy, , can be obtained from

where $H$ is the total enthalpy of the corresponding system, and indicates the vacancy in an $N$-site

supercell. The solute-vacancy binding energy, , is defined as

where $\mathrm{X}$ indicates the substitutional element in an $\mathrm{N}$-site supercell.

\section{COMPUTATIONAL METHODOLOGY}

$A b$ initio calculations are used in this work to determine lattice constants, defect formation enthalpies, migration barriers, binding enthalpies, and vibrational frequencies for the Ni self-diffusion and $\mathrm{Ag}$ impurity diffusion coefficients in a pure fcc Ni system. All calculations were performed using the projector augmented-wave (PAW) method [11] as implemented in the Vienna ab initio simulation package (VASP) [12-13] with the provided GW PAW potentials [14]. The potentials used treat all semicore states as valence. The generalized gradient approximation (GGA) with Perdew-Burke-Ernzerhof (PBE) [15] parametrization was utilized for the exchange-correlation functional.

We employed supercells with 32 lattice sites (2x2x2 conventional fcc cells), replacing one host atom with $\mathrm{Ag}$ as the impurity atom, or removing one host atom to form a vacancy. The energy cutoff for the plane wave basis was $500 \mathrm{eV}$. The Brillouin zone was sampled with a Monkhorst-Pack $k$ point mesh of $12 \times 12 \times 12$. All degrees of freedom of the crystal structures were allowed to relax, including cell shape, cell volume, and atomic positions using the conjugate gradient algorithm as implemented in the VASP code, until the forces on all unconstrained atoms were $\leq 0.01 \mathrm{eV} / \AA$. The energy convergence in self- 
consistent field calculations was chosen to be $10^{-5} \mathrm{eV}$. All calculations were spin polarized and allowed for magnetic ordering. Migration barriers were determined by the nudged elastic band method (NEB) [16]. Vibrational frequencies were determined via the calculation of the interatomic force constants, using density functional perturbation theory (DFPT) as implemented in VASP 5.4.

\section{RESULTS AND DISCUSSION}

The methods outlined in Section 2 have been used to calculate vacancy-mediated Ni-self and Ag diffusion coefficients in pure fcc Ni. The parameters needed for vacancy-mediated impurity diffusion are summarized in Table 1. All values are determined using ab initio methods, and all optimized structures are ferromagnetic.

Table 1. Calculated properties from current PBE calculations to, in comparison with, experimental data and other theoretical calculations.

\begin{tabular}{|c|c|c|c|c|}
\hline Property & System & Present work & Experimental data & $\begin{array}{l}\text { Other } \\
\text { computations }\end{array}$ \\
\hline $\mathrm{a}(\AA)$ & fcc Ni & 3.50 & $\begin{array}{l}3.5238[17] \\
3.5157[18]\end{array}$ & 3.5239 [19] \\
\hline$\Delta \mathrm{H}_{\mathrm{f}}(\mathrm{eV})$ & vacancy & 1.48 & $\begin{array}{l}1.4[5] \\
1.73[6] \\
1.56[7]\end{array}$ & $\begin{array}{l}1.40-1.45[3] \\
1.63-1.81[4]\end{array}$ \\
\hline$\Delta \mathrm{H}_{\mathrm{m}}(\mathrm{eV})$ & vacancy & 1.19 & $\begin{array}{l}1.5[5] \\
1.04[6]\end{array}$ & \\
\hline $\begin{array}{l}\mathrm{Q}(\mathrm{eV}) \\
\left(\Delta \mathrm{H}_{\mathrm{f}}+\Delta \mathrm{H}_{\mathrm{m}}\right)\end{array}$ & vacancy & 2.67 & $\begin{array}{l}2.90[5] \\
2.81[20] \\
2.77[6]\end{array}$ & $2.62-3.03[4]$ \\
\hline $\begin{array}{l}-\Delta \mathrm{H}_{\mathrm{b}}{ }^{\mathrm{Ag}-\mathrm{V}}(\mathrm{eV}) \\
\Delta \mathrm{H}_{\mathrm{f}}^{\mathrm{V}}-\Delta \mathrm{H}_{\mathrm{b}}{ }^{\mathrm{Ag}-\mathrm{V}}(\mathrm{eV}) \\
\Delta \mathrm{H}_{\mathrm{m}}{ }^{\mathrm{Ag} / \mathrm{Ni}}(\mathrm{eV}) \\
\mathrm{Q}^{\mathrm{Ag} / \mathrm{Ni}}(\mathrm{eV})\end{array}$ & $\begin{array}{l}\text { Ag-V } \\
\text { Ag-V } \\
\text { Ag-V } \\
\text { Ag-V }\end{array}$ & $\begin{array}{l}-0.283 \\
1.197 \\
0.732 \\
1.929\end{array}$ & $2.047[5]$ & \\
\hline & vacancy & 11.272 & & \\
\hline$(\mathrm{THz})$ & & & & \\
\hline$(\mathrm{THz})$ & Ag-V & 1.053 & & \\
\hline & vacancy & $\mathrm{N} / \mathrm{A}$ & 3.3 [7] & 1.82 [19] \\
\hline
\end{tabular}

$\left(\kappa_{\mathrm{B}}\right)$ 


\begin{tabular}{|c|c|c|c|c|}
\hline$\left(\kappa_{\mathrm{B}}\right)$ & $\mathrm{Ag}-\mathrm{V}$ & N/A & & \\
\hline $\mathrm{D}_{0}\left(\mathrm{~cm}^{2} / \mathrm{sec}\right)$ & vacancy & 0.0666 & $\begin{array}{l}1.9[5] \\
0.534[20]\end{array}$ & $0.0444-0.0745[4]$ \\
\hline $\mathrm{D}_{0}\left(\mathrm{~cm}^{2} / \mathrm{sec}\right)$ & $\mathrm{Ag}-\mathrm{V}$ & 0.0062 & $0.025[5]$ & \\
\hline
\end{tabular}

\subsection{Lattice Parameter}

Table 1 lists the lattice parameter, . The lattice parameter for pure Ni has been experimentally

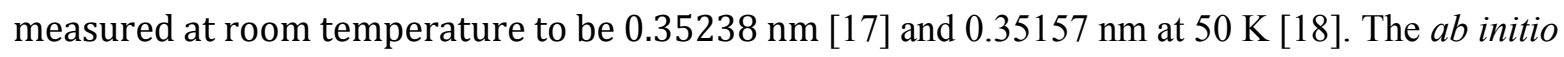

calculated value used in this work is $0.35 \mathrm{~nm}(0.35239 \mathrm{~nm}$ [19]), which is considered to be at $0 \mathrm{~K}$. Since

the models are appropriate when there is only a dilute amount of solute present ( $\sim 1 \%$ or less $)$, the pure $\mathrm{Ni}$

lattice constant is used for all tracer diffusion calculations. The $a b$ initio calculated lattice parameter value will be used in order to be consistent with the other $a b$ initio determined values.

\subsection{Thermodynamic Properties}

In order to calculate the concentration of thermally created vacancy defects, the enthalpy of formation must be determined. The calculations of enthalpy of formation and the solute-vacancy binding energy are

more or less routine nowadays; however, the evaluation of the vibrational entropy changes $S_{\mathrm{f}}$ and $S_{\mathrm{b}}$ is

non-trivial due to problems obtaining converged values, which seems to require a very large effort to

adjust DFT parameters. In this report, we have assumed values of $1.82 k_{\mathrm{B}}[19]$ and $0.0 k_{\mathrm{B}}$ for , and $0.0 k_{\mathrm{B}}$

for , respectively. We are currently still performing actual calculations of these due to vibrational

contributions.

The thermodynamic properties of the initial and transition states of a jump need to be determined to

Equation (4). Each initial state is completely relaxed

calculate its effective jump frequency, , using 
with respect to internal coordinates, the volume, and shape. We quantitatively determine the transition state with the saddle point along the minimum energy diffusion path by NEB [16] calculations. Conducting NEB calculations is specifically important for the case of jumps with asymmetry between the initial and final states.

Table 1 illustrates our calculated enthalpy of vacancy formation and migration barrier in pure fcc

$\mathrm{Ni}$, as well as the enthalpy of solute-vacancy binding ( $\Delta$ ) and migration barrier () of Ag in Ni. Where

available, these quantities are compared with experimental measurements. We find a good

agreement between our first-principles-calculated energetics and experimentally measured quantities.

The corresponding Arrhenius parameters are presented in Table 1, along with the results from a previous DFT study [4], the Zhang mobility assessment [20] and selected single-crystal experimental data [5-6]. Table 1 shows that in the present work, the activation energies for self-diffusion and vacancy-

mediated Ag diffusion, , are within 10\% ( $<6 \%$ for Ag-Ni) of the single-crystal experimental data. In

general, the data appears lower than the prediction for finite temperature calculations. Similar trends are

observed for the diffusion prefactor, .

Note that our DFT calculations were performed at $0 \mathrm{~K}$. There is clearly a discrepancy between the high-temperature (closer to the melting temperature) estimates from experiments and low-temperature approximations from DFT. We suspect that this discrepancy with experimental data may be due to the use of non-equilibrium quenching methods to obtain the experimental values [4], a large scatter in the data at lower temperatures, and a strong non-Arrhenius behavior in measurements close to the melting temperature [3], in contrast with the $\mathrm{T}=0 \mathrm{~K}$ ab initio calculations.

\subsection{Diffusion Coefficients}

After Arrhenius diffusion data were determined within the GGA and DFPT, the diffusion coefficients were calculated according to Equation (1). Figure 1 shows our prediction results for self-diffusion and vacancy-mediated $\mathrm{Ag}$ diffusion in pure fcc $\mathrm{Ni}$ for two vacancy-formation vibrational entropies of $1.8 k_{\mathrm{B}}$ and $0 k_{\mathrm{B}}$, respectively, alongside experimental data. Prediction of diffusion coefficients is one of the most 
stringent tests of the ability for DFT calculations, because of the complex interplay of lattice constant, vacancy formation enthalpy, solute-vacancy binding enthalpy, and vibrational frequencies in these supercell calculations including defects. As shown in the Arrhenius plot of $D$ versus 1000/T in Figure 1, the accuracy in general is quite impressive, given the expected accuracy of current DFPT calculations, especially with the value of $1.82 k_{B}$ for the vacancy formation vibrational entropy adopted from previous DFT calculations [19]. In fact, the discrepancy is only significant in the case where the vacancy formation vibrational entropy is not taken into account, in which case the prediction is about half an order of magnitude lower than the experimental data at low temperatures, and about an order of magnitude lower at high temperatures.

With, the most significant discrepancies with the experimental data are underestimation by about

0.05 orders of magnitude at low temperatures for Ni self-diffusion, as well as overestimation by about 0.06 orders of magnitude at high temperatures for Ag diffusion. Even there, the discrepancy might be attributed to the fact that experimental data only exists in the higher-temperature paramagnetic regime above the Curie temperature of $\mathrm{Ni}$ around $625 \mathrm{~K}$, and our DFPT calculations are at $0 \mathrm{~K}$ with spinpolarized and magnetic ordering leading to a ferromagnetic phase. In fact, nickel is ferromagnetic up below its Curie temperature, at which point it transforms to paramagnetic phase. This makes direct, quantitative comparison with experimental data very difficult. In addition, the discrepancy may also result from the small vacancy-formation entropy change due to vibrational frequencies at different temperature.

Previous first-principles studies of diffusion coefficients in the literature have used either the Debye model or the phonon supercell approach [4] for calculating finite temperature thermodynamic properties. Particularly at lower temperatures, the results on the Arrhenius plots in general underestimate the experimental diffusion data in all cases, and the most significant underestimation was about an order of magnitude. At high temperature, the underestimation is about half an order of magnitude. Consistent with other observations in the literature, the use of the phonon supercell approach (i.e., Debye model or quasiharmonic approximation) for the calculation of finite temperature thermodynamic properties within the local density approximation (LDA) and lower energy cutoff results in an underestimation of the diffusion coefficient with respect to experimental data. Apparently, present work using GGA-derived exchange and correlation functionals with the DFPT approach yields much better results [3].






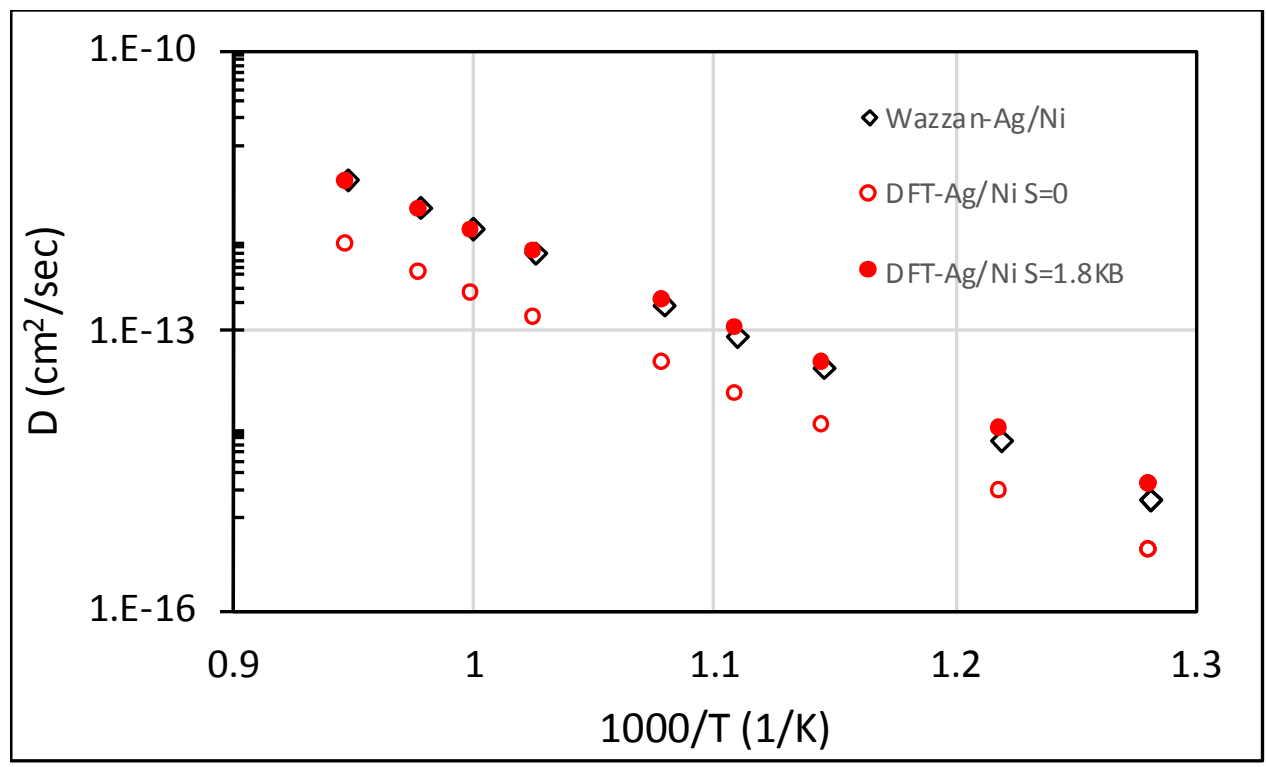

Figure 1. Coefficients of self-diffusion (top) and vacancy-mediated Ag diffusion (bottom) in pure fcc Ni for two vacancy formation vibrational entropies of $1.8 k_{\mathrm{B}}$ and $0 k_{\mathrm{B}}$, respectively, compared with experimental data.

\section{RESULTS AND DISCUSSION}

We have illustrated how impurity diffusion coefficients can be predicted directly from first principles without any empirical or fitting parameters. Specifically, we have used DFT static (energy) and DFPT (vibrational) calculations to obtain lattice constants, defect formation enthalpies, migration barriers, vacancy-solute binding enthalpies, vibrational frequencies, and the impurity-jump frequency within the framework of transition state theory. Prediction of diffusion coefficients is one of the most stringent tests of the ability of DFT calculations, because of the complex interplay of lattice constant, vacancy formation enthalpy, solute-vacancy binding enthalpy, and vibrational frequencies.

Using the DFPT approach, we were able to obtain the values of the individual diffusion parameters

along with the diffusion pre-factor and activation energy for Ni self-diffusion and Ag diffusion in

pure fcc Ni. The results match well with the experimental data. Both the diffusion prefactor and the

activation energy are key to a quantitative description of vacancy-mediated self-diffusion or solute diffusivities. It is also found that even the qualitative trends of diffusivity in fcc Ni may not always be inferred from activation energies or migration barriers alone. Especially given some limitations of the proposed methods (i.e., that all of the experimental data that exists is in the paramagnetic regime, while the calculations were performed in the ferromagnetic regime), the calculations provide very impressive agreement with experimentally measured diffusion coefficients for $\mathrm{Ag}$ in fcc nickel.

The present work shows that the DFPT approach may be a viable means to determine Ag diffusion coefficients accurately in Ni alloys. We are currently still performing actual calculations of entropies due to vibrational contributions, since the evaluation of 
the vibrational entropy changes $S_{\mathrm{f}}$ and $S_{\mathrm{b}}$ is non-trivial. Future plans to utilize this DFPT approach would

extend initially to Ag diffusion in binary nickel alloys such as $\mathrm{NiMo}, \mathrm{NiCr}$ and $\mathrm{NiFe}$ and ternary $\mathrm{Ni}$ alloys such as $\mathrm{NiCrFe}$ and $\mathrm{NiCrMo}$. The ultimate goal is a predictive tool for determination of $\mathrm{Ag}$ diffusion coefficients in Ni super-alloys such as Inconel 713C, and Hastelloy X, or other prospective turbine blade materials.

\section{REFERENCES}

[1] Milman, V., et al., Free energy and entropy of diffusion by ab initio molecular dynamics: Alkali ions in silicon, Physical Review Letters, 1993,Vol. 70(19): pp. 2928-2931.

[2] Ke, J.-H., G.A. Young, and J.D. Tucker, Ab initio study of phosphorus effect on vacancymediated process in nickel alloys - An insight into Ni2Cr ordering, Acta Materialia, 2019, Vol. 172, pp. 30-43.

[3] Gong, Y., et al., Temperature dependence of the Gibbs energy of vacancy formation of fcc Ni, Physical Review B, 2018, Vol. 97(21), pp. 214106.

[4] Hargather, C.Z., et al., A first-principles study of self-diffusion coefficients of fcc Ni. Computational Materials Science, 2014, Vol. 86, pp. 17-23.

[5] Wazzan, A.R., P. Tung, and L.B. Robinson, Diffusion of Silver into Nickel Single Crystals. Journal of Applied Physics, 1971., Vol. 42(13), pp. 5316-5320.

[6] Wolff, J., et al., Vacancy formation in nickel and $\alpha$-nickel-carbon alloy. Acta Materialia, 1997., Vol. 45(11), pp. 4759-4764.

[7] Scholz, H.-P., Messungen der absoluten leerstellenkonzentration in nickel und geordneten intermetallischen nickel-legierungen mit einem differentialdilatometer, Ph.D. thesis, University of Göttingen. 2001.

[8] Mehrer, H., Diffusion in Solids: Fundamentals, Methods, Materials, Diffusion-controlled Processes, 2007, Springer.

[9] Claire, A.D.L., in Treatise on Physical Chemistry (Academic Press, New York, 1970), Vol. 10, pp. 261.

[10] Vineyard, G.H., Frequency factors and isotope effects in solid state rate processes. Journal of Physics and Chemistry of Solids, 1957, Vol. 3(1), pp. 121-127.

[11] Blochl, P.E., Projector Augmented-Wave Method. Physical Review B, 1994, Vol. 50(24), pp. 17953-17979.

[12] Kresse, G. and J. Furthmüller, Efficient iterative schemes for ab initio total-energy calculations using a plane-wave basis set, Physical Review B, 1996, Vol. 54(16), pp. 11169-11186.

[13] Kresse, G. and J. Furthmüller, Efficiency of ab-initio total energy calculations for metals and 
semiconductors using a plane-wave basis set, Computational Materials Science, 1996, Vol. 6(1), pp. $15-50$.

[14] Kresse, G. and D. Joubert, From ultrasoft pseudopotentials to the projector augmented-wave method. Physical Review B, 1999, Vol. 59(3), pp. 1758-1775.

[15] Perdew, J.P., K. Burke, and M. Ernzerhof, Generalized Gradient Approximation Made Simple. Physical Review Letters, 1996, Vol. 77(18), pp. 3865-3868.

[16] Mills, G., H. Jónsson, and G.K. Schenter, Reversible work transition state theory: application to dissociative adsorption of hydrogen, Surface Science, 1995, Vol. 324(2), pp. 305-337.

[17] Ehrhart, P., in Atomic Defects in Metals, Vol. 25, Springer-Verlag, Berlin, New York 1991, pp. 88.

[18] Bandyopadhyay, J. and K.P. Gupta, Low temperature lattice parameter of nickel and some nickelcobalt alloys and Grüneisen parameter of nickel, Cryogenics, 1977, Vol. 17(6): pp. 345-347.

[19] Tucker, J.D., et al., Ab initio-based diffusion theory and tracer diffusion in $\mathrm{Ni}-\mathrm{Cr}$ and $\mathrm{Ni}-\mathrm{Fe}$ alloys, Journal of Nuclear Materials, 2010, Vol. 405(3), pp. 216-234.

[20] Zhang, L., et al., Atomic mobilities and diffusivities in the fcc, L12 and B2 phases of the Ni-Al system, International Journal of Materials Research, 2010, Vol. 101(12), pp. 1461-1475. 